\title{
NANOTECNOLOGÍA APLICADA EN MATERIALES REFRACTARIOS: UNA REVISIÓN
}

\section{NANOTECHNOLOGY APPLIED IN REFRACTORY MATERIALS:}

\section{A REVIEW}

\section{Marco Vinicio Carrión V. ${ }^{1 *}$ \& Fernanda Pilaquinga F. ${ }^{1,2}$}

Recibido: 19 de marzo 2020 / Aceptado: 2 de junio 2020 /

Publicado en línea: 8 de junio de 2020

DOI: $10.26807 / \mathrm{ia.v8i} 2.141$

Palabras claves: materiales refractarios, nanomateriales, grafeno, titanio, óxido férrico, magnesia, zirconia, sílice y alúmina

Keywords: refractory materials, nanomaterials, graphene, titanium, ferric oxide, magnesia, zirconia, alumina, silica

\section{RESUMEN}

Los materiales refractarios son de gran importancia para la humanidad, debido a su capacidad de resistir altas temperaturas. Son empleados en la industria del cemento, cerámica, vidrio, metalúrgica, siderúrgica y petroquímica. La mayor preocupación a nivel industrial es que los materiales refractarios son sometidos a varios mecanismos de desgaste durante el proceso de producción, tales como:

1 Universidad Internacional SEK, Maestría en Diseño Industrial y Procesos, Quito, Ecuador $\left({ }^{*}\right.$ correspondencia: mvcarrion.mdin@uisek.edu.ec; maria.pilaquinga@uisek.edu.ec)

2 Pontificia Universidad Católica del Ecuador, Facultad de Ciencias Exactas y Naturales, Escuela de Ciencias Químicas, Quito, Ecuador (mfpilaquingaf@puce.edu.ec) 
ataque químico, mecánico, termomecánico y carga térmica. Si el material no cuenta con las propiedades adecuadas, su durabilidad se verá afectada, y por ende los costos de producción. En este sentido la combinación de los nanomateriales, se convierte en una importante alternativa para mejorar las propiedades de los materiales refractarios, proporcionándoles mejor densidad, baja porosidad, resistencia a la erosión, baja conductividad térmica, entre otras. El presente artículo realiza una revisión de varios trabajos de investigación, acerca de la evolución de los nanomateriales aplicados a los materiales refractarios, entre los que se mencionan el uso de grafeno, dióxido de titanio, óxido férrico, magnesia, zirconia, alúmina y sílice, para mejorar las propiedades físicas de los materiales refractarios. De manera general, los estudios demuestran que el uso de los nanomateriales, provee a los materiales refractarios mejores propiedades, en rangos de concentración específicos. Sin embargo, todos los estudios han sido realizados a nivel de laboratorio, por lo que sería promisorio su uso a nivel industrial.

\section{ABSTRACT}

Refractory materials are of great importance for humanity, due to their ability to resist high temperatures. They are used in cement, ceramics, glass, metallurgical, steelmaker, and petrochemical industry. The biggest concern at the industrial level is that the refractory materials are subjected to various wear mechanisms during the production process, such as chemical, mechanical, thermomechanical, and thermal loading. If the material does not have the properties, its durability will be affected, and therefore the production costs. In this way the combination of the nanomaterials, becomes an important alternative to improve the properties of the refractory materials providing them with a better density, low porosity, resistance to erosion, low thermal conductivity, among others. The present article makes a review of several research papers about the evolution of the nanomaterials applied on the refractories, including the use of graphene, titanium dioxide, ferric oxide, magnesia, zirconia, alumina, and silica, to improve the physical properties of the refractory materials. In general, studies showed that the use of nanomaterials provides to the refractories better properties, in a specific concentration ranges. However, all the studies have been carried out at the laboratory level so their use at the industrial level would be promising. 


\section{INTRODUCCIÓN}

Los materiales refractarios son ampliamente utilizados, y en grandes volúmenes en las industrias del acero, aluminio, cemento, vidrio, siderúrgica y petroquímica (Dehsheikh, Ghasemi-Kahrizsangi, Karamian, \& Nemati, 2019). La búsqueda para mejorar las propiedades mecánicas de los materiales refractarios, relacionada con los costos de producción, ha visto en la nanotecnología una alternativa. La nanotecnología es considerada como la principal iniciativa tecnológica a nivel industrial (Al-Nemrawi, AbuAlSamen, \& Alzoubi, 2019). La nanotecnología permite obtener nuevos materiales con mayor rendimiento y durabilidad (Alivisatos, 1996). Los nanomateriales inorgánicos se presentan con mayor interés, como materia prima de nuevos materiales (Chandra, Kumari, Bontempi, \& Yadav, 2020; Jamkhande, Ghule, Bamer, \& Kalaskar, 2019). Es por ello que en presente estudio, se analizan investigaciones recientes respecto al uso de diferentes nanomateriales, para mejorar las propiedades mecánicas de los materiales refractarios, empleados a nivel industrial.
Los materiales refractarios se definen como compuestos inorgánicos "no metálicos", que pueden soportar ambientes de trabajo con temperaturas superiores a los $538{ }^{\circ} \mathrm{C}$, sin sufrir alteraciones en sus propiedades físicas o químicas (Roy, Chandra, \& Maitra, 2019). Este tipo de materiales, químicamente inertes, presentan un elevado punto de fusión debido a las fuertes interacciones de sus enlaces iónicos y covalentes. Sin embargo, son frágiles a temperatura ambiente y presentan una baja resistencia a la tensión (Hancock, Homfray, Porton, Todd, \& Wynne, 2018). La industria del acero, hierro, cemento, y vidrio emplea el uso de refractarios como parte importante en el recubrimiento de reactores, su estructura es policristalina y sus propiedades dependen de su microestructura (Moreno, 2008). Los materiales refractarios tienen una composición definida, se clasifican como: refractarios de arcilla, sílice, básicos y especiales. En la Tabla 1, se presenta la composición química de diferentes materiales refractarios comerciales (William D. Callister, 2002). 
El comportamiento de los materiales refractarios depende principalmente de su composición y su uso puede variar dependiendo de la necesidad industrial. Los sectores industriales donde se emplea los materiales refractarios son (Güeto, 2010):

- Cemento: hornos rotativos, intercambiadores de calor y enfriadores.

- Cerámica: hornos túnel y de cámara

- Vidrio: hornos de fusión y temple, y baños de estaño.

- Acero: hornos eléctricos, desgasificación y laminación.

- Metalúrgica: hornos de fusión de cobre y aluminio, y torres fusoras.

- Siderúrgica: altos hornos, estufas, baterías de horno cok y mezclador de arrabio.
- Petroquímica: Hornos de nafta y cracking.

Es evidente que gran parte de los materiales refractarios son utilizados como revestimiento de hornos, ya que permiten mantener las temperaturas de operación estables. El sector industrial que más emplea materiales refractarios, es la siderúrgica con un $60 \%$. La elevada demanda de este tipo de materiales, ha generado una rápida evolución en los últimos años, debido a las exigencias impuestas por los nuevos procesos industriales, que han provocado un mayor control de materias primas, mejoramiento en los procesos fabricación e investigaciones en las propiedades fisicoquímicas de los materiales (Fu, Gu, Huang, Zhang, \& Wu, 2020).

Tabla 1. Composición de refractarios comerciales (William D. Callister, 2002)

\begin{tabular}{cccccccc}
\hline Tipos de refractario & \multicolumn{7}{c}{ Composición (\%p/p) } \\
& $\mathrm{TiO}_{2}$ & $\mathrm{Fe}_{2} \mathrm{O}_{3}$ & $\mathbf{M g O}$ & $\mathrm{SiO}_{2}$ & $\mathrm{Al}_{2} \mathrm{O}_{3}$ & $\mathrm{CaO}$ & $\mathrm{Cr}_{2} \mathrm{O}_{3}$ \\
\hline $\begin{array}{c}\text { Arcilla refractaria } \\
\text { Arcilla refractaria con alto } \\
\text { contenido de alúmina }\end{array}$ & $1-2$ & $0-1$ & $0-1$ & $70-50$ & $25-45$ & $0-1$ & \\
$\begin{array}{c}\text { Periclasa } \\
\text { Sílice }\end{array}$ & $1-4$ & $0-1$ & $0-1$ & $10-45$ & & $0-1$ & \\
& & 3 & 90 & 3 & 1 & 2,5 & 0,3 \\
\hline
\end{tabular}


El uso de la nanotecnología aplicada a los materiales refractarios, inició en el año 1999 (Khoroshavin \& Perepelitsyn, 1999). La evolución histórica de las publicaciones referentes al desarrollo de los nanomateriales en aplicaciones refractarias, revela una tendencia creciente en el tiempo, obtenida de la página Web of Science (WoS), tal como se muestran en la Figura1.

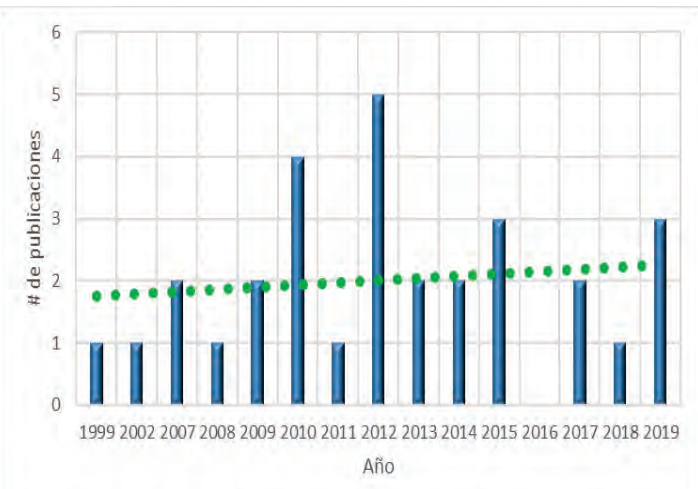

Figura 1. Evolución histórica de publicaciones de nanomateriales en refractarios

(Wos, Clarivative Analytics)

Desde otra óptica, el estudio de materiales refractarios evidencia que los países asiáticos lideran el desarrollo de los nanomateriales en este campo, y Ilama la atención la mínima participación de países como: Italia, Reino Unido, y, Estados Unidos, como se muestra en la Figura 2.

Los diferentes autores utilizan un nanomaterial específico, para mejorar las propiedades de un material re- fractario base, generalmente compuesto por óxido de magnesio o también conocido como magnesia $(\mathrm{MgO})$, dióxido de silicio o sílice $\left(\mathrm{SiO}_{2}\right)$, y óxido de aluminio o alúmina $\left(\mathrm{Al}_{2} \mathrm{O}_{3}\right)$. Los nanomateriales que generalmente se usan para mejorar las propiedades de los materiales refractarios son: nanopartículas de grafeno, dióxido de titanio, óxido férrico, magnesia, zirconia, alúmina y sílice. 


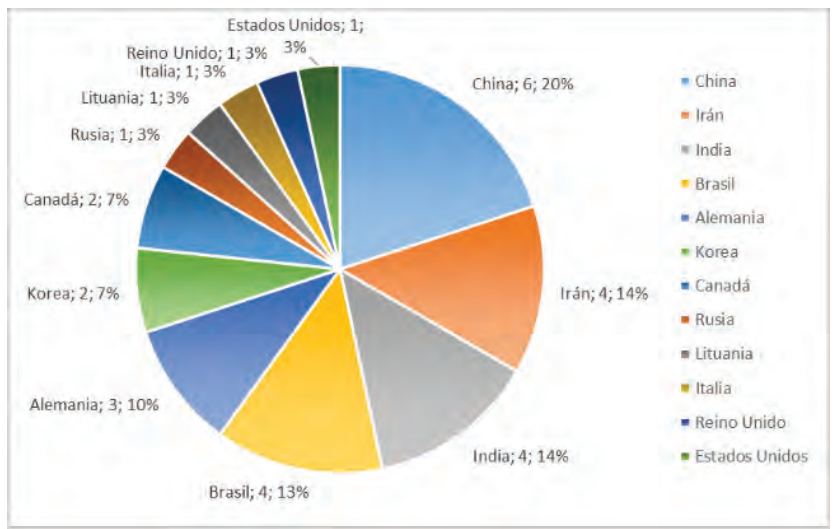

Figura 2. Artículos científicos publicados por país

\section{Grafeno}

El grafeno es un material constituido por pequeñas partículas de carbono, agrupadas en forma de láminas muy finas que forman celdas hexagonales (Pham et al., 2020). En la Figura 3, se observa la disposición espacial de los átomos de grafeno.
Este material es único, ya que cada átomo de carbono está unido a otro por medio de una nube electrónica. El grafeno, se obtiene de una fuente natural, como es el grafito, empleado en industrias tales como la: automotriz, telefónica, aeronáutica, informática, entre otras (X. Li \& Zhi, 2018; Pham et al., 2020; Wu et al., 2020).
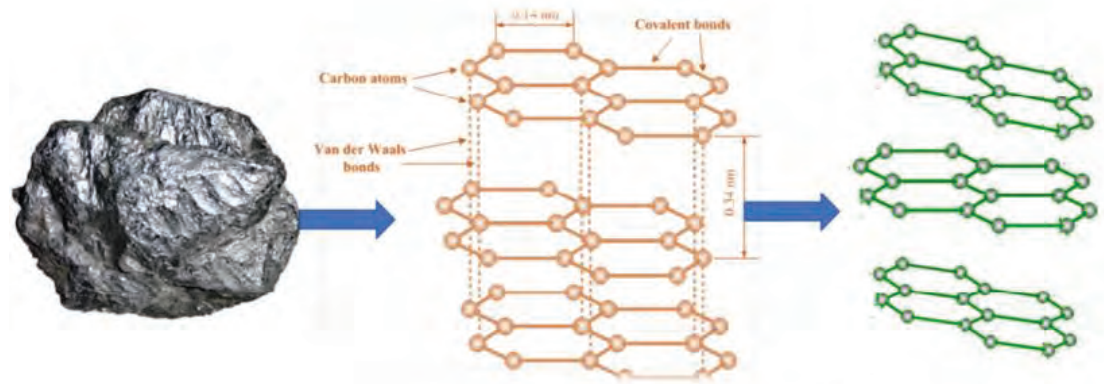

Figura 3. Disposición espacial del grafeno

(Tiwari, Sahoo, NannanWang, \& Huczko, 2020) 
El grafeno presenta una elevada área en relación a su volumen (Al-Nemrawi et al., 2019). El grafeno prístino, tiene un área de $2630 \mathrm{~m}^{2} / \mathrm{g}$, el grafeno negro de humo 850-900 y los nanotubos de carbono 100-1000 $\mathrm{m}^{2} / \mathrm{g}$ respectivamente.

El grafeno en combinación con materiales refractarios, mejora su tenacidad y resistencia (García, 2016).

\section{Nanopartículas de dióxido de Titanio} ( $\mathrm{TiO} 2)$

El dióxido de titanio $\left(\mathrm{TiO}_{2}\right)$ es un mineral natural que se puede encontrar en forma de rutilo, anatasa y brookita (Figura 4). Cuando las estructuras de la anatasa y la brookita son sometidas a calor, éstas se convierten en rutilo, que es una estructura más estable a un rango más amplio de temperaturas.
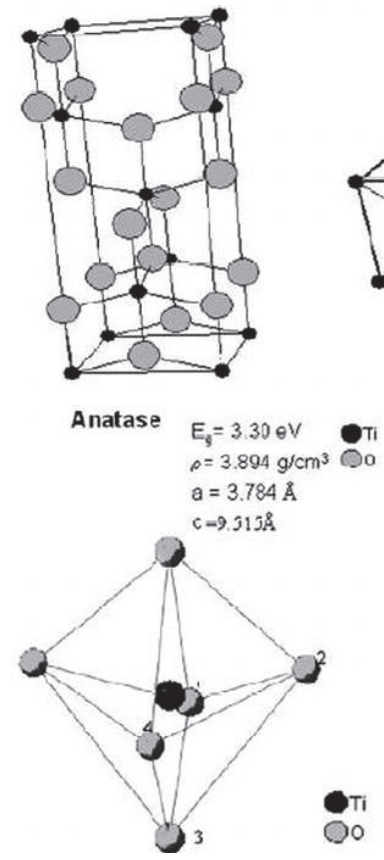

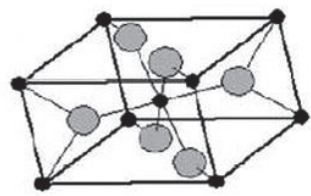

Rutile

$\mathrm{Eg}=3.05 \mathrm{eV}$ $\rho=4.250 \mathrm{gcm}^{3}$ $\mathrm{a}=4.593 \mathrm{~A}$ $c=2.959 A$

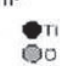

Brookite

Ti.O, $d_{n p}=1980 \AA, d_{n}=1934 \mathrm{~A}$ $01-02=2.80 \mathrm{~A}$ $02-03=304 A$ O3 $-.04=2.47 \AA$

Figura 4. Minerales de $\mathrm{TiO}_{2}$

(Nyamukamba, Okoh, Mungondori, Taziwa, \& Zinya, 2018) 
$\mathrm{El} \mathrm{TiO}_{2}$ presenta propiedades elevadas de índice de refracción, no es fotodegradable, es químicamente inerte y presenta estabilidad térmica (H. Kim et al., 2019). El rutilo, por ejemplo, tiene un índice de refracción de 2, 6 a 2,8; punto de fusión de $1825{ }^{\circ} \mathrm{C}$ y punto de ebullición de 2500 a $3000{ }^{\circ} \mathrm{C}$. Las nanopartículas de $\mathrm{TiO} 2$ aprovechan y repotencian las propiedades del material macro. Su propiedad fotocatalítica se emplea en celdas solares, semiconductores, tratamiento de aguas contaminadas, etc. (Thakur, Kumar, \& Kumar, 2019).

\section{Nanopartículas de óxido férrico} $\left(\mathrm{Fe}_{2} \mathrm{O}_{3}\right)$

Las nanopartículas de óxido férrico $\left(\mathrm{Fe}_{2} \mathrm{O}_{3}\right)$, presentan interesantes pro- piedades magnéticas (Al-Nemrawi et al., 2019; Bouafia \& Laouini, 2020). Presentan tamaños menores a 100 nm y una forma esférica, además de una elevada capacidad de reacción por intercambio iónico, adsorción o precipitación iónica. En la Figura 5, se muestra el núcleo de una nanopartícula de hierro. Se puede observar que el núcleo, presenta hierro con valencia cero, mientras que en la superficie tiene iones de diferente número de oxidación. Las nanopartículas de $\mathrm{Fe}_{2} \mathrm{O}_{3}$ en materiales refractarios, mejoran la dureza, resistencia térmica y eléctrica (X. Q. Li, Elliott, \& Zhang, 2006).

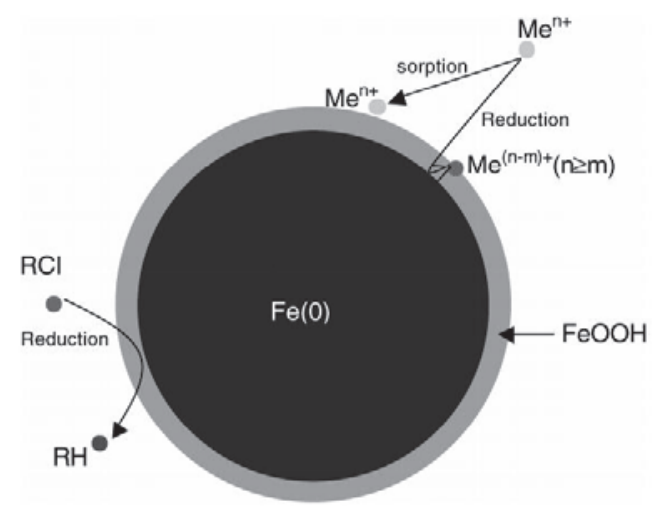

Figura 5. Nanopartículas de hierro

(X. Q. Li et al., 2006) 
En la Figura 6, se puede apreciar la imgen obtenida de un microscopio de transmisión electrónica (TEM, por sus siglas en inglés) de las nanopartículas de $\mathrm{Fe}_{2} \mathrm{O}_{3}$.

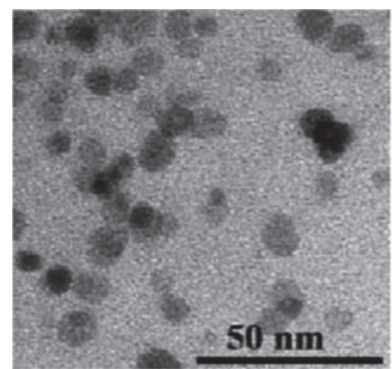

Figura 6. Imagen TEM de nanopartículas de $\mathrm{Fe}_{2} \mathrm{O}_{3}$

(Menon et al., 2017)

\section{Nanopartículas de magnesia (MgO)}

Los materiales de magnesia convencionales poseen muy buenas propiedades refractarias, tales como alta resistencia a la compresión en frío (400-800 kg/ $\left.\mathrm{cm}^{2}\right)$ y a temperatura elevada $\left(1500{ }^{\circ} \mathrm{C}\right.$, bajo carga de $\left.2 \mathrm{~kg} / \mathrm{cm}^{2}\right)$, y alta resistencia al ataque en medio básico (Alvarez, Criado, \& Baudin, 1992).

Las nanopartículas de óxido de magnesio $(\mathrm{MgO})$ tienen tamaño entre 5070 nm (Bhattacharya, Swain, Giri, \& Neogi, 2019). Se pueden emplear en electrónica, catálisis, cerámica, productos petroquímicos, recubrimientos y muchos otros campos (Zou et al., 2014).

En la Figura 7, se puede apreciar la imagen TEM de nanopartículas de zinc con nanopartículas de óxido de magnesio como refractario.

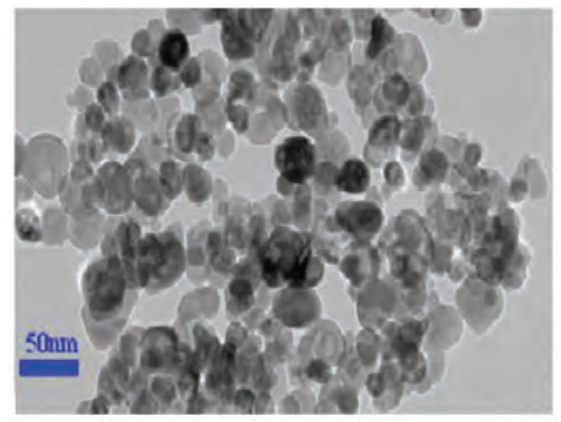

Figura 7. Material refractario de nanopartículas de zinc con $\mathrm{MgO}$ (Ghasemi-Kahrizsangi, Barati Sedeh, Gheisari Dehsheikh, Shahraki, \& Farooghi, 2016)

\section{Nanopartículas de zirconia $\left(\mathrm{ZrO}_{2}\right)$}

El óxido de zirconio o zirconia $\left(\mathrm{ZrO}_{2}\right)$, es un material con diferentes estructuras cristalinas dependiendo de la temperatura, esto quiere decir que es polimórfico (Zakaria et al., 2019). La zirconia, es de elevada resistencia mecánica y a la fracturación (Serve, Boreave, Cartoixa, Pajot, 
\& Vernoux, 2019). Además, presenta elevada resistencia a la corrosión, excelentes propiedades eléctricas, es químicamente inerte y gran estabilidad térmica (Roberts, Dodson, Carpinone, \& Hagelin-Weaver, 2015; Sigwadi, Mokrani, \& Dhlamini, 2019). En la Figura 8, se muestra las estructuras cristalinas que del $\mathrm{ZrO}_{2}$ : monoclínica, tetragonal y cúbica.

a)
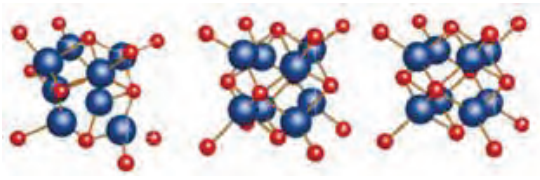

Figura 8. Estructuras del $\mathrm{ZnO}_{2}$ a) monoclínica, b) tetragonal y c) cúbica (Andrade-Guel, Cabello-Alvarado, \& Ávila-Orta, 2019)

La estructura monoclínica del $\mathrm{ZnO}_{2}$ es generada a la temperatura ambiental hasta los $1170{ }^{\circ} \mathrm{C}$, la tetragonal se obtiene en el rango de $1170-2370{ }^{\circ} \mathrm{C}$ y la cúbica a temperaturas de $2650^{\circ} \mathrm{C}$. Muchos materiales cerámicos están basados en el $\mathrm{ZnO}_{2}$, ya que beneficia las propiedades de dureza, térmicas y químicas (Efaw et al., 2020).

\section{Nanopartículas de alúmina $\left(\mathrm{Al}_{2} \mathrm{O}_{3}\right)$}

Las nanopartículas de óxido de aluminio o alúmina $\left(\mathrm{Al}_{2} \mathrm{O}_{3}\right)$, son consi- deradas como un material prometedor para el desarrollo de nuevas estructuras a nivel industrial (B. Kim, Song, Kim, Hwang, \& Park, 2019). Este nanomaterial presenta algunas ventajas como: elevada resistencia y dureza, alto punto de fusión, resistencia a la corrosión y capacidad aislante (Sezavar, Zebarjad, \& Sajjadi, 2015). La estabilidad mecánica de la alúmina es un factor importante para el desarrollo de nuevas estructuras, ya que proporciona alta compactibilidad y resistencia a la contracción (Khalilpourazary \& Salehi, 2019). Los materiales que se usan como soporte para las nanopartículas $\mathrm{Al}_{2} \mathrm{O}_{3}$, mejoran la estabilidad térmica, reducen su desgaste y aumentan el nivel de aislamiento térmico. (Khalilpourazary \& Salehi, 2019). En la Figura 9, se puede observar la imagen TEM de las nanopartículas de $\mathrm{Al}_{2} \mathrm{O}_{3}$.

Este tipo de nanopartículas han Ilamado la atención de los científicos debido a sus prometedoras propiedades físicas y químicas, resaltando su biocompatibilidad, resistencia mecánica, estabilidad química y su versatilidad para ser sintetizadas (Zamani, Jafari, Mousavii, \& Darezereshki, 2020). 


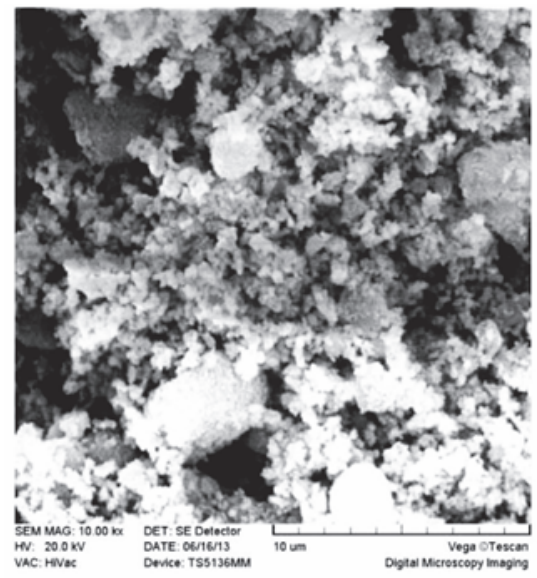

Figura 9. Imagen TEM de las nanopartículas de $\mathrm{Al}_{2} \mathrm{O}_{3}$ (Khalilpourazary \& Salehi, 2019)

\section{Nanopartículas de sílice $\left(\mathrm{SiO}_{2}\right)$}

Estos nanomateriales se caracterizan por tener un soporte de sílice con poros entre un rango de tamaño de 2 a $50 \mathrm{~nm}$, generando una superficie externa y otra al interior de los poros, de allí su versatilidad para ser utilizada en el campo de la biotecnología y la ciencia de materiales (Morales, Castán, Ortega, \& Ruiz, 2019). A continuación, en la Figura 10, se presenta la estructura y una micrografía de las nanopartículas de sílice mesoporosa.
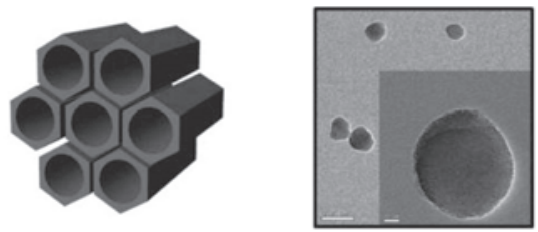

Figura 10. Nanopartículas de sílice mesoporosa (Morales et al., 2019)

\section{RESULTADOS}

Los resultados de los estudios realizados, conducen a una tendencia creciente del uso de nanopartículas en materiales refractarios. En su análisis, se corroboran las diferencias de comportamiento a nivel macro y na- nométrico. En la Tabla 2, se muestran los diferentes tipos de nanomateriales que se han empleado en materiales refractarios, para mejorar diferentes propiedades físicas, mecánicas, térmicas o termomecánicas. 


\section{Tabla 2. Nanomateriales empleados en materiales refractarios}

\begin{tabular}{|c|c|c|c|c|}
\hline $\begin{array}{c}\text { Tipo de } \\
\text { nanopartícula }\end{array}$ & Matriz & Propiedad mejorada & Limitación & Referencias \\
\hline \multirow[t]{3}{*}{ Grafeno } & $\mathrm{MgO}-\mathrm{C}$ & Refractariedad & & $\begin{array}{c}\text { (Bag, Adak, } \\
\text { \& Sarkar, 2012) }\end{array}$ \\
\hline & & $\begin{array}{l}\text { Dispersabilidad } \\
\text { Resistencia a la } \\
\text { oxidación }\end{array}$ & & $\begin{array}{l}\text { (Wang et al., 2014) } \\
\text { (W. Kim, Oh, } \\
\text { \& Shon, 2015) } \\
\text { (Kumar, Singh, } \\
\text { \& Datta, 2017) }\end{array}$ \\
\hline & & Moldeabilidad & & $\begin{array}{l}\text { (Liu, Yan, Reece, } \\
\text { \& Jiang, 2012) }\end{array}$ \\
\hline $\mathrm{TiO}_{2}$ & $\mathrm{MgO}-\mathrm{C}$ & $\begin{array}{c}\text { Resistencia a la } \\
\text { oxidación }\end{array}$ & & $\begin{array}{l}\text { (Aneziris, Hubálková, } \\
\text { \& Barabás, 2007) } \\
\text { (Manivasakan et al., } \\
\text { 2010) }\end{array}$ \\
\hline $\mathrm{SiO}_{2}$ & & $\begin{array}{c}\text { Densidad aparente } \\
\text { Expansión térmica } \\
\text { reversible }\end{array}$ & & (Carlucci et al., 2015) \\
\hline $\mathrm{Fe}_{2} \mathrm{O}_{3}$ & $\mathrm{Mg}-\mathrm{Cr}$ & Sinterizado & & $\begin{array}{l}\text { (Azhari, Golestani- } \\
\text { Fard, \& Sarpoolaky, } \\
\text { 2009) }\end{array}$ \\
\hline \multirow[t]{2}{*}{$\mathrm{Al}_{2} \mathrm{O}_{3}$} & $\mathrm{Al}_{2} \mathrm{O}_{3}-\mathrm{C}$ & $\begin{array}{c}\text { Esfuerzo mecánico } \\
\text { Resistencia choque } \\
\text { térmico }\end{array}$ & & $\begin{array}{c}\text { (Roungos \& Aneziris, 2012) } \\
\text { (Ghasemi-Kahrizsangi, } \\
\text { Gheisari Dehsheikh, } \\
\text { Karamian, Ghasemi- } \\
\text { Kahrizsangi, \& Vahid } \\
\text { Hosseini, 2017) }\end{array}$ \\
\hline & Bauxita & Cristalización & $\begin{array}{l}\text { Reduce } \\
\text { la densidad } \\
\text { aparente }\end{array}$ & \\
\hline $\begin{array}{l}\text { Coloides a base } \\
\text { de } \mathrm{Al}_{2} \mathrm{O}_{3} \text { y } \mathrm{SiO}_{2}\end{array}$ & & $\begin{array}{c}\text { Secado rápido } \\
\text { Sinterizado } \\
\text { Estabilidad volumétrica } \\
\text { Aglomeración } \\
\text { Esfuerzo mecánico } \\
\text { Refractariedad }\end{array}$ & & $\begin{array}{l}\text { (Nouri-Khezrabad, } \\
\text { Braulio Pandolfelli, } \\
\text { Golestani-Fard, } \\
\text { \& Rezaie, 2013) }\end{array}$ \\
\hline
\end{tabular}

Respecto al uso de grafeno, Bag et al. (2012), desarrollaron el estudio del comportamiento de los refractarios de magnesia carbón $\mathrm{MgO}-\mathrm{C}$, en presencia de nanopartículas de grafeno. Encontraron que su adición permite 
reducir la oxidación del carbono de la matriz refractaria, lo que a su vez se refleja en un mejor aislamiento térmico del proceso metalúrgico (metalurgia primaria en horno de arco eléctrico, metalurgia secundaria en horno cuchara), reduciendo las pérdidas de calor en el metal líquido, y, finalmente, favoreciendo a la reducción del consumo de energía eléctrica, que representa alrededor del $28 \%$ de los costos de transformación del acero y el $56 \%$ en su balance energético.

Las nanopartículas de grafeno (Wang et al., 2014) presentan una alta dispersabilidad en la matriz cerámica de los refractarios de alúmina, y, a su vez, aceleran la formación de las ramificaciones del cristal cerámico.

Kim et al. (2015), en su investigación acerca de como mejorar la resistencia a la oxidación de un refractario de magnesia carbón MgO-C, determinaron que al lograr inhibir o reducir la oxidación del carbono, se podía reducir la velocidad de oxidación del material refractario en general. Para este proceso emplearon un recubrimiento de nanopartículas de grafeno. La función del recubri- miento con grafeno, es su reacción con el oxígeno y así evitar la oxidación del carbono presente en el material refractario. En la Figura 11, se muestran imágenes comparativas entre los grados de oxidación de un refractario $\mathrm{MgO}-\mathrm{C}$ sin recubrimiento (a), y con recubrimiento de grafeno (b).

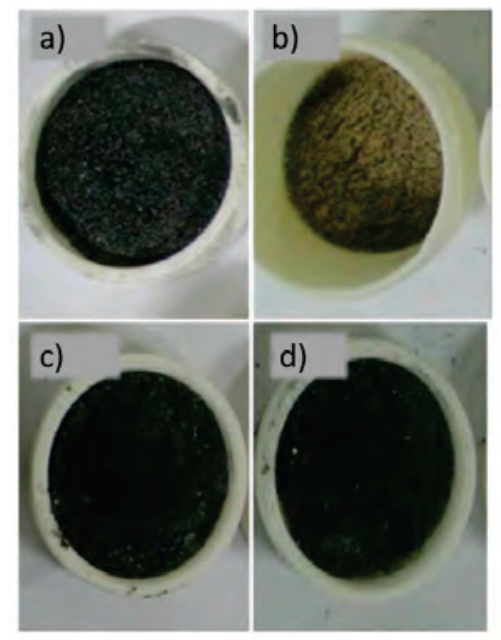

Figura 11. Material refractario de $\mathrm{MgO}-\mathrm{C}$ sin partículas de grafeno ( $a$ y b), y con partículas de recubrimiento (b y d) (E. H. Kim, Cho, Lim, Byeun, \& Jung, 2015)

Adicionalmente, respecto a las nanopartículas de grafeno, se ha desarroIlado un estudio para mejorar las propiedades de moldeabilidad de los concretos monolíticos, debido a este 
nanomaterial puede humectarse en agua (Kumar et al., 2017). En la Figura 12 , se muestran los de humectabilidad del grafeno a diferentes concentraciones. Liu et al. (2012); respecto al uso del grafeno en materiales refractarios, determinaron que este nanomaterial presenta excelente dispersión en la matriz cerámica, y, además, no presenta daños al ser sometido a la temperatura de sinterización.

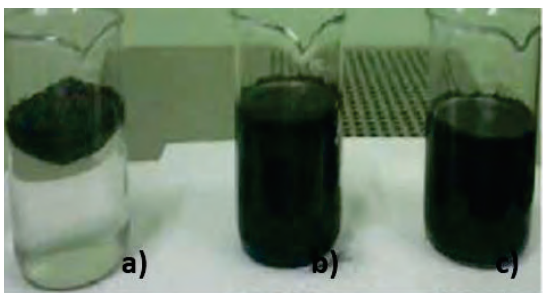

Figura 12. Grafito natural (a), $10 \%$ grafeno (b) y $15 \%$ grafeno (c)

(Kumar et al., 2017)

Al analizar el uso de nanopartículas de $\mathrm{TiO}_{2}$, Aneziris et al. (2007), desarrollaron un trabajo de investigación acerca de la influencia en la microestructura en ladrillos refractarios de magnesia y carbón (MgO-C), modificado con partículas de $\mathrm{TiO}_{2}$. Su principal aporte fue la mejora en la resistencia a la oxidación. Además, determinaron que a medida que dis- minuye el tamaño a nanopartícula de $\mathrm{TiO}_{2}$, esta propiedad mejora significativamente. En la Figura 13, se muestra un ladrillo refractario de MgO-C con nanopartículas de $\mathrm{TiO}_{2}$ (A) y sin nanopartículas (B).
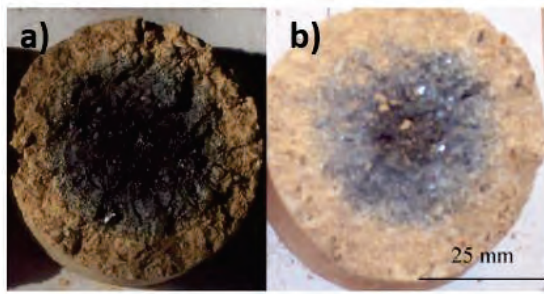

Figura 13. Ladrillo refractario de MgO-C (a) con nanopartículas de $\mathrm{TiO}_{2}$ y (b) sin nanopartículas

Aneziris et al., 2007)

Manivasakan y Venkatachalaman (2010), emplearon nanopartículas de $\mathrm{TiO}_{2}$ a partir de mineral ilmenita proveniente de la India, y las aplicaron en ladrillos refractarios de sílice, obteniendo como resultado principal una mejora en la densidad aparente, lo cual incide directamente en la reversibilidad de su expansión térmica. En la Tabla 3, se muestran la variación de las propiedades con la aplicación de las nanopartículas de $\mathrm{TiO}_{2}$, sobre un ladrillo refractario de sílice. 
Tabla 3. Propiedades con/sin nano $\mathrm{TiO}_{2}$ (Manivasakan y Venkatachalaman, 2010)

\begin{tabular}{lcc}
\hline Parámetro & \multicolumn{2}{c}{ Ladrillo de sílice } \\
& Sin nano $\mathrm{TiO}_{2}$ & Con nano $\mathrm{TiO}_{2}$ \\
\hline $\begin{array}{l}\text { Densidad } \\
\text { aparente } \\
\left(\mathrm{Kg} / \mathrm{m}^{3}\right)\end{array}$ & $1,80+/-0,10$ & $1,92+/-0,01$ \\
\hline $\begin{array}{l}\text { Expansión } \\
\text { térmica } \\
\text { reversible } \\
\text { a } 1000^{\circ} \mathrm{C}\end{array}$ & $1,35+/-0,01$ & $1,15+/-0,01$ \\
\hline
\end{tabular}

Carlucci et al. (2015) aportaron al estudio del efecto del $\mathrm{TiO}_{2}$ en la matriz refractaria de sílice, indicando que disminuye la porosidad del material. En la Figura 14, se muestran cuatro micrografías obtenida por microscopía electrónica de barrido (SEM, por sus siglas en inglés), donde se pueden apreciar las diferencias entre el material sin nanopartículas de $\mathrm{TiO}_{2}$ que presenta porosidades y grietas (A y C), y el material modificado (B y D), a diferentes escalas de medida (500 y $200 \mu \mathrm{m}$ respectivamente).

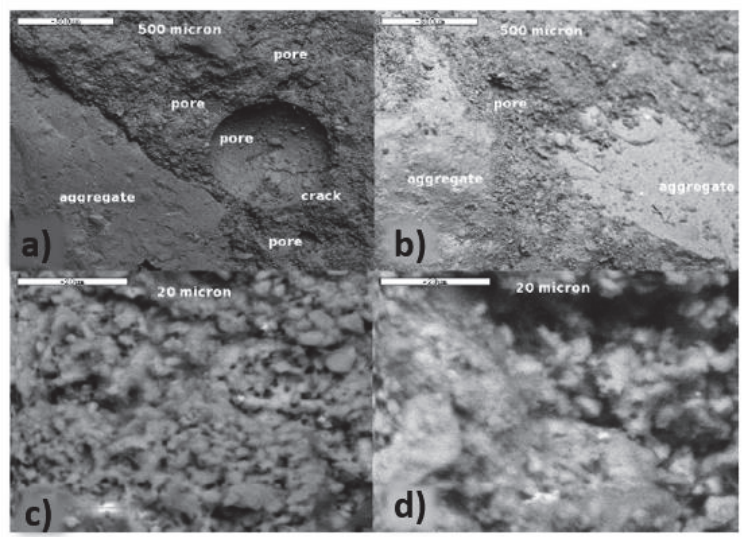

Figura 14. Micrografías SEM de un material refractario de sílice (a y b) sin partículas y con partículas de $\mathrm{TiO}_{2}$ (b y c).

(Carlucci et al., 2015)

Usando nanopartículas de $\mathrm{Fe}_{2} \mathrm{O}_{3}$, Azhari et al. (2009) estudiaron el efecto de la inclusión de nanopartículas de óxidos de hierro en la microestruc- tura de una matriz de ladrillos refractarios magnesia-cromo Mg-Cr. El estudio encontró que la presencia de nanopartículas de óxido férrico 
$\left(\mathrm{Fe}_{2} \mathrm{O}_{3}\right)$, favorece a la formación de espinela magnesio ferrítica $\left(\mathrm{MgAl}_{2} \mathrm{O}_{4}\right)$ a bajas temperaturas, mejorando el proceso de sinterizado de la matriz del material refractario.

Por otro lado, al emplear nanopartículas de MgO, Braulio et al. (2008), estudiaron los mecanismos de expansión de la espinela de magnesia $\left(\mathrm{MgAl}_{2} \mathrm{O}_{4}\right)$ en un cuerpo refractario, a través de la adición de nanopartículas de $\mathrm{MgO}$, con el objetivo de: anticipar la temperatura de reacción que da inicio a la formación de la espinela, y a la vez reducir su expansión, brindando mejores características térmicas al material. La adición de las nanopartículas de $\mathrm{MgO}$ debe ser máximo del $3 \%$ en peso, debido a que en mayores cantidades no se encuentran beneficios en términos del control de la expansión de la espinela.

Al utilizar nanopartículas de $\mathrm{ZrO}_{2}$, Chen et al. (2007) realizaron un estudio de las propiedades de una matriz refractaria de magnesia calcita $\mathrm{MgO}$ $\mathrm{CaO}$ sinterizando polvos de zirconita $\mathrm{ZrO}_{2}$ a $1600{ }^{\circ} \mathrm{C}$. El estudio evidenció un notable incremento en la densificación de la matriz MgO-CaO, cuando el tamaño de las partículas de $\mathrm{ZrO}_{2}$ fue llevado a escala nano. En la Figura 15, se muestra una comparación entre el peso de $\mathrm{ZrO}_{2}$ (\%) vs la densidad aparente y el porcentaje de porosidad. Claramente se puede observar que la densidad aparente se incrementa con el porcentaje en peso de $\mathrm{ZrO}_{2}$, mientras que la porosidad tiene un comportamiento inverso, siendo mayor el efecto cuando el tamaño de la partícula es a escala nano.

Con nanopartículas de alúmina $\mathrm{Al}_{2} \mathrm{O}_{3}$, Roungos y Aneziris (2012) estudiaron el comportamiento de las nanopartículas de polvo de alúmina, espinela y carbón en una matriz refractaria de alúmina y carbón $\mathrm{Al}_{2} \mathrm{O}_{3}-\mathrm{C}$, y determinaron que existe una mejora en el esfuerzo mecánico tanto a temperatura ambiente como a $1400{ }^{\circ} \mathrm{C}$ (temperatura de trabajo), así como también en la resistencia al choque térmico.

Ghasemi et al. (2017), en su estudio referente al efecto de las nanopartículas de $\mathrm{Al}_{2} \mathrm{O}_{3}$, al ser incorporadas en un concreto de bajo cemento de matriz bauxítica, modifican sus propiedades físicas y mecánicas. Determinaron que la adición de las nano- 


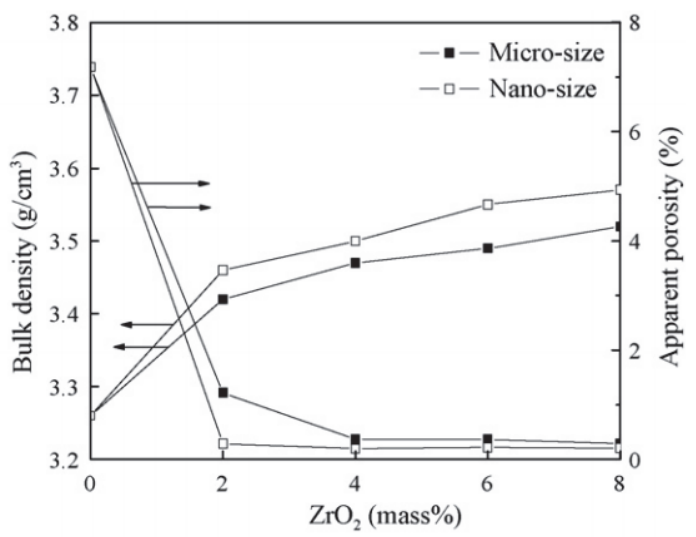

Figura 15. Porcentaje (\%p/p) de $\mathrm{ZrO}_{2}$ vs densidad aparente y porosidad (Chen, Lu, \& YU, 2007)

partículas de $\mathrm{Al}_{2} \mathrm{O}_{3}$ en cantidades inferiores a $1 \%$ en peso, favorecen la formación de compuestos cristalinos planos y tipo aguja, que se incrustan en los límites de grano de las partículas de bauxita, mejorando sus propiedades como aislante térmico, así como, su resistencia mecánica. Sin embargo, el efecto negativo se vio en la reducción de la densidad aparente del material.

Finalmente, Khezrabad et al. (2013), estudiaron el uso de la alúmina y sílica nanométrica coloidal, como ele- mentos ligantes en reemplazo del cemento calcio aluminato. Luego de la investigación se llegó a determinar que el uso de los coloides de sílica y alumina mejoran propiedaes como: secado rápido, alta sinterabilidad, estabilidad volumétrica, aglomeración, esfuerzo mecánico, y propiedades termomecánicas a altas temperaturas. En la Figura 16, se muestran ejemplos de los perfiles de erosión de muestras refractarias con: cemento calcio aluminato, alúmina hidratable, nanosílica coloidal y nanoalúmina coloidal. 

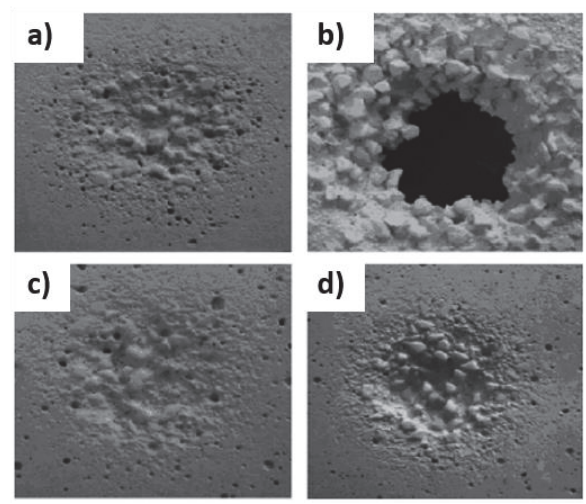

Figura 16. Erosión de cemento calcio aluminato sin nanopartículas coloidales (a y b) y con nanopartículas de sílice y alúmina (c y d) (Ghasemi-Kahrizsangi et al., 2017).

Se puede observar que las muestras con suspensiones nanocoloidales presentan una menor erosión.

\section{CONCLUSIÓN}

El uso de nanomateriales para mejorar las propiedades físicas, mecánicas, térmicas y termomecánicas de los materiales refractarios, se ha podido notar en el resultado de las investigaciones analizadas. Es importante considerar la proporción de la mezcla del material y nanomaterial, ya que cuando se superan los valores límites, algunas de las propiedades se ven afectadas y por lo tanto las características del material final. Todos los estudios han sido llevados a cabo a nivel de laboratorio, y sería un gran aporte para la industria, que se tome como base el desarrollo de las publicaciones científicas.

\section{AGRADECIMIENTOS}

Este estudio fue realizado como proyecto final de la asignatura de Nanotecnologías y Materiales Funcionales para Diseño, de la Maestría en Dise- ño Industrial y de Procesos de la Universidad Internacional SEK del Ecuador, a cargo de la Ph. D. (c) Fernanda Pilaquinga. 


\section{LISTA DE REFERENCIAS}

Al-Nemrawi, N. K., AbuAlSamen, M. M., \& Alzoubi, K. H. (2019). Awareness about nanotechnology and its applications in drug industry among pharmacy students. Currents in Pharmacy Teaching and Learning, 0-1. https://doi.org/10.1016/j. cptl.2019.12.003

Alivisatos, A. P. (1996). Semiconductor clusters, nanocrystals, and quantum dots. Science, 271(5251), 933-937. https://doi.org/10.1126/science.271.5251.933

Alvarez, C., Criado, E., \& Baudin, C. (1992). Refractarios de magnesia-grafito. In BOL. SOC. ESP. CERAM. VIDR (Vol. 31).

Andrade-Guel, M. L., Cabello-Alvarado, C. J., \& Ávila-Orta, C. A. (2019). Dióxido de zirconio: alternativas de síntesis y aplicaciones biomédicas. Retrieved February 9, 2020, from CienciaUAT website: http://www.revistaciencia.uat.edu.mx/index.php/ CienciaUAT/article/view/1152/591

Aneziris, C. G., Hubálková, J., \& Barabás, R. (2007). Microstructure evaluation of MgO$\mathrm{C}$ refractories with $\mathrm{TiO} 2-$ and $\mathrm{Al}$-additions. Journal of the European Ceramic Society, 27(1), 73-78. https://doi.org/10.1016/j.jeurceramsoc.2006.03.001

Azhari, A., Golestani-Fard, F., \& Sarpoolaky, H. (2009). Effect of nano iron oxide as an additive on phase and microstructural evolution of Mag-Chrome refractory matrix. Journal of the European Ceramic Society, 29(13), 2679-2684. https://doi.org/10. 1016/j.jeurceramsoc.2009.03.032

Bag, M., Adak, S., \& Sarkar, R. (2012). Study on low carbon containing MgO-C refractory: Use of nano carbon. Ceramics International, 38(3), 2339-2346. https://doi.org/10.1016/j.ceramint.2011.10.086

Bhattacharya, P., Swain, S., Giri, L., \& Neogi, S. (2019). Fabrication of magnesium oxide nanoparticles by solvent alteration and their bactericidal applications. Journal of Materials Chemistry B, 7(26), 4141-4152. https://doi.org/10.1039/c9tb00782b 
Bouafia, A., \& Laouini, S. E. (2020). Green synthesis of iron oxide nanoparticles by aqueous leaves extract of Mentha Pulegium L.: Effect of ferric chloride concentration on the type of product. Materials Letters, 265, 127364. https://doi.org/ 10.1016/j.matlet.2020.127364

Carlucci, C., Conciauro, F., Scremin, B. F., Antico, A. G., Muscogiuri, M., Sibillano, T., ... Ciccarella, G. (2015). Properties of aluminosilicate refractories with synthesized boron-modified TiO 2 nanocrystals. Nanomaterials and Nanotechnology, 5(1), 1 7. https://doi.org/10.5772/60204

Chandra, H., Kumari, P., Bontempi, E., \& Yadav, S. (2020). Medicinal plants: Treasure trove for green synthesis of metallic nanoparticles and their biomedical applications. Biocatalysis and Agricultural Biotechnology, 101518. https://doi.org /10.1016/j.bcab.2020.101518

Chen, M., Lu, C., \& Yu, J. (2007). Improvement in performance of MgO-CaO refractories by addition of nano-sized ZrO2. Journal of the European Ceramic Society, 27(16), 4633-4638. https://doi.org/10.1016/j.jeurceramsoc.2007.04.001

Dehsheikh, H. G., Ghasemi-Kahrizsangi, S., Karamian, E., \& Nemati, A. (2019). Recent Advancement in monolithic refractories via application of Nanotechnology "A review Paper". Journal of Nanoanalysis, 6(1), 1-20. https://doi.org/10.22034 /jna.2019.664385

Efaw, C. M., Vandegrift, J. L., Reynolds, M., Jaques, B. J., Hu, H., Xiong, H., \& Hurley, M. F. (2020). Characterization of zirconium oxides part II: New insights on the growth of zirconia revealed through complementary high-resolution mapping techniques. Corrosion Science, 108491. https://doi.org/10.1016/j.corsci.2020. 108491

Fu, L., Gu, H., Huang, A., Zhang, M., \& Wu, J. (2020). Fabrication of CaO-MgO-Al2O3 materials from metallurgical waste industrial residue and their potential usage in MgO-C refractories. Ceramics International, 46(1), 959-967. https://doi.org/10. 1016/j.ceramint.2019.09.057

García, D. (2016). Estudio del daño por contacto de materiales compuesto cerámica/ grafeno. 
Ghasemi-Kahrizsangi, S., Barati Sedeh, M., Gheisari Dehsheikh, H., Shahraki, A., \& Farooghi, M. (2016). Densification and properties of ZrO2 nanoparticles added magnesia-doloma refractories. Ceramics International, 42(14), 15658-15663. https://doi.org/10.1016/j.ceramint.2016.07.021

Ghasemi-Kahrizsangi, S., Gheisari Dehsheikh, H., Karamian, E., Ghasemi-Kahrizsangi, A., \& Vahid Hosseini, S. (2017). The influence of Al2O3 nanoparticles addition on the microstructure and properties of bauxite self-flowing low-cement castables. Ceramics International, 43(12), 8813-8818. https://doi.org/10.1016/j.ceramint. 2017.04.013

Güeto, J. M. (2010). Tecnología de los materiales cerámicos. Retrieved February 8, 2020, from Google Libros website: https://books.google.com.ec/books?id=4H6OXg N1w6wC\&printsec $=$ frontcover $\& d q=$ material + refractarios + pdf $\&$ hl $=$ es 419\&sa=X\&ved=0ahUKEwiLueeUscPnAhWro1kKHUJIAb8Q6AEIMjAB\#v=onepage \&q\&f=false

Hancock, D., Homfray, D., Porton, M., Todd, I., \& Wynne, B. (2018). Refractory metals as structural materials for fusion high heat flux components. Journal of Nuclear Materials, 512, 169-183. https://doi.org/10.1016/j.jnucmat.2018.09.052

Jamkhande, P. G., Ghule, N. W., Bamer, A. H., \& Kalaskar, M. G. (2019, October 1). Metal nanoparticles synthesis: An overview on methods of preparation, advantages and disadvantages, and applications. Journal of Drug Delivery Science and Technology, Vol. 53. https://doi.org/10.1016/j.jddst.2019.101174

Khalilpourazary, S., \& Salehi, J. (2019). How alumina nanoparticles impact surface characteristics of Al7175 in roller burnishing process. Journal of Manufacturing Processes, 39, 1-11. https://doi.org/10.1016/j.jmapro.2019.01.027

Khoroshavin, L. B., \& Perepelitsyn, V. A. (1999). On the nanotechnology of refractories. Refractories and Industrial Ceramics, 40(11-12), 553-557. https://doi.org/10. 1007/BF02762640

Kim, B., Song, J., Kim, J. Y., Hwang, J., \& Park, D. (2019). The control of particle size distribution for fabricated alumina nanoparticles using a thermophoretic separator. Advanced Powder Technology, 30(10), 2094-2100. https://doi.org/10.1016/ j.apt.2019.06.023 
Kim, E. H., Cho, G. H., Lim, H. T., Byeun, Y. K., \& Jung, Y. G. (2015). Development of $\mathrm{MgO}-\mathrm{C}$ refractory having high oxidation resistance by metal coating process. Journal of Nanoscience and Nanotechnology, 15(1), 514-517. https://doi.org/ 10.1166/jnn.2015.8338

Kim, H., Jeon, D., Oh, S., Nam, K. S., Son, S., Chan Gye, M., \& Shin, I. (2019). Titanium dioxide nanoparticles induce apoptosis by interfering with EGFR signaling in human breast cancer cells. Environmental Research, 175, 117-123. https://doi.org/10.1016/j.envres.2019.05.001

Kim, W., Oh, H. S., \& Shon, I. J. (2015). The effect of graphene reinforcement on the mechanical properties of $\mathrm{Al} 2 \mathrm{O} 3$ ceramics rapidly sintered by high-frequency induction heating. International Journal of Refractory Metals and Hard Materials, 48, 376-381. https://doi.org/10.1016/j.ijrmhm.2014.10.011

Kumar, K., Singh, R. K., \& Datta, R. (2017). Water wettable graphite through nanotechnology and its application in refractories. InterCeram: International Ceramic Review, 66(1-2), 30-35. https://doi.org/10.1007/bf03401199

Li, X. Q., Elliott, D. W., \& Zhang, W. X. (2006). Zero-valent iron nanoparticles for abatement of environmental pollutants: Materials and engineering aspects. Critical Reviews in Solid State and Materials Sciences, 31(4), 111-122. https://doi.org/ 10.1080/10408430601057611

Li, X., \& Zhi, L. (2018, May 7). Graphene hybridization for energy storage applications. Chemical Society Reviews, Vol. 47, pp. 3189-3216. https://doi.org/10.1039/ c7cs00871f

Liu, J., Yan, H., Reece, M. J., \& Jiang, K. (2012). Toughening of zirconia/alumina composites by the addition of graphene platelets. Journal of the European Ceramic Society, 32(16), 4185-4193. https://doi.org/10.1016/j.jeurceramsoc.2012.07.007

Manivasakan, P., Rajendran, V., Rauta, P. R., Sahu, B. B., Sahu, P., Panda, B. K., ... Jegadesan, S. (2010). Effect of TiO2 nanoparticles on properties of silica refractory. Journal of the American Ceramic Society, 93(8), 2236-2243. https://doi.org/ 10.1111/j.1551-2916.2010.03727.x 
Menon, P. K., Sharma, A., Lafuente, J. V., Muresanu, D. F., Aguilar, Z. P., Wang, Y. A., ... Sharma, H. S. (2017). Intravenous Administration of Functionalized Magnetic Iron Oxide Nanoparticles Does Not Induce CNS Injury in the Rat: Influence of Spinal Cord Trauma and Cerebrolysin Treatment. In International Review of Neurobiology (Vol. 137, pp. 47-63). https://doi.org/10.1016/bs.irn.2017.08.005

Morales, M. E., Castán, H., Ortega, E., \& Ruiz, M. A. (2019). Silica Nanoparticles: Preparation, Characterization and Applications in Biomedicine. Pharmaceutical Chemistry Journal, 53(4), 329-336. https://doi.org/10.1007/s11094-019-02001-3

Moreno, L. M. V. (2008). Materiales industriales. Teoría y aplicaciones. Retrieved February 8, 2020, from Google Libros website: https://books.google.com.ec/ books?id=VSdtMx8Oj8wC\&pg=PA131\&dq=material+refractarios+pdf\&hl=es419\&sa=X\&ved=0ahUKEwiLueeUscPnAhWro1kKHUJIAb8Q6AEIOzAC\#v=onepage $\& q=$ material refractarios $p d f \& f=f a l s e$

Nouri-Khezrabad, M., Braulio, M. A. L., Pandolfelli, V. C., Golestani-Fard, F., \& Rezaie, H. R. (2013). Nano-bonded refractory castables. Ceramics International, 39(4), 3479-3497. https://doi.org/10.1016/j.ceramint.2012.11.028

Nyamukamba, P., Okoh, O., Mungondori, H., Taziwa, R., \& Zinya, S. (2018). Synthetic Methods for Titanium Dioxide Nanoparticles: A Review. In Titanium Dioxide - Material for a Sustainable Environment. https://doi.org/10.5772/intechopen.75425

Pham, M. H., Khazaeli, A., Godbille-Cardona, G., Truica-Marasescu, F., Peppley, B., \& Barz, D. P. J. (2020). Printing of graphene supercapacitors with enhanced capacitances induced by a leavening agent. Journal of Energy Storage, 28. https://doi.org/10.1016/j.est.2020.101210

Roberts, S. J., Dodson, J. J., Carpinone, P. L., \& Hagelin-Weaver, H. E. (2015). Evaluation of nanoparticle zirconia supports in the thermochemical water splitting cycle over iron oxides. International Journal of Hydrogen Energy, 40(46), 15972-15984. https://doi.org/10.1016/j.ijhydene.2015.09.109

Roungos, V., \& Aneziris, C. G. (2012). Improved thermal shock performance of Al 2 O 3$\mathrm{C}$ refractories due to nanoscaled additives. Ceramics International, 38(2), 919927. https://doi.org/10.1016/j.ceramint.2011.08.011 
Roy, J., Chandra, S., \& Maitra, S. (2019). Nanotechnology in castable refractory. Ceramics International, 45(1), 19-29. https://doi.org/10.1016/j.ceramint.2018.09.261

Serve, A., Boreave, A., Cartoixa, B., Pajot, K., \& Vernoux, P. (2019). Synergy between Ag nanoparticles and yttria-stabilized zirconia for soot oxidation. Applied Catalysis B: Environmental, 242, 140-149. https://doi.org/10.1016/j.apcatb.2018.09.069

Sezavar, A., Zebarjad, S., \& Sajjadi, S. (2015). A Study on the Effect of Nano Alumina Particles on Fracture Behavior of PMMA. Technologies, 3(2), 94-102. https://doi.org/10.3390/technologies3020094

Sigwadi, R., Mokrani, T., \& Dhlamini, M. (2019). The synthesis, characterization and electrochemical study of zirconia oxide nanoparticles for fuel cell application. Physica B: Condensed Matter. https://doi.org/10.1016/j.physb.2019.411842

Thakur, B. K., Kumar, A., \& Kumar, D. (2019). Green synthesis of titanium dioxide nanoparticles using Azadirachta indica leaf extract and evaluation of their antibacterial activity. South African Journal of Botany, 124, 223-227. https://doi.org/10.1016/ j.sajb.2019.05.024

Tiwari, S. K., Sahoo, S., NannanWang, \& Huczko, A. (2020). Graphene Research and their Outputs: Status and Prospect. Journal of Science: Advanced Materials and Devices. https://doi.org/10.1016/j.jsamd.2020.01.006

Wang, Q., Li, Y., Luo, M., Sang, S., Zhu, T., \& Zhao, L. (2014). Strengthening mechanism of graphene oxide nanosheets for Al 2O3-C refractories. Ceramics International, 40(1 PART A), 163-172. https://doi.org/10.1016/j.ceramint.2013.05.117

William D. Callister. (2002). Introducción a la ciencia e ingeniería de los materiales. Retrieved February 8, 2020, from Google libros website: https://books.google.com.ec /books?id=gnfPV1txXiUC\&pg=PA445\&dq=MATERIAL+REFRACTARIOS\&hl=es419\&sa $=$ X\&ved=0ahUKEwjWmZSdhsDnAhWRnFkKHaLjB50Q6AEIMTAB\#v=on epage $\& q \& f=$ false

Wu, D. Y., Zhou, W. H., He, L. Y., Tang, H. Y., Xu, X. H., Ouyang, Q. S., \& Shao, J. J. (2020). Micro-corrugated graphene sheet enabled high-performance all-solid-state film supercapacitor. Carbon, 160, 156-163. https://doi.org/10.1016 /j.carbon. 2020.01.019 